\title{
Evidence for the formation of metallic In after laser irradiation of InP
}

Cite as: J. Appl. Phys. 126, 025902 (2019); https://doi.org/10.1063/1.5109230

Submitted: 07 May 2019. Accepted: 21 June 2019. Published Online: 12 July 2019

L. Cabral (D), J. Andrés (D), T. R. Machado (D), A. Picinin (D), J. P. Rino (D), V. Lopez-Richard (D), E. Longo (iD), A. F. Gouveia (D) G. E. Marques (D), E. Z. da Silva (D), and M. A. San-Miguel (D)
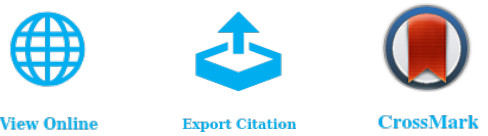

View Online

Export Citation

\section{ARTICLES YOU MAY BE INTERESTED IN}

Imaging an isolated water molecule using a single electron wave packet

The Journal of Chemical Physics 151, 024306 (2019); https://doi.org/10.1063/1.5100520

Upconversion emission studies of $\mathrm{Tm}^{3+} / \mathrm{Yb}^{3+}$ doped $\mathrm{MgWO}_{4}$ for blue emitting devices AIP Conference Proceedings 2115, 030164 (2019); https://doi.org/10.1063/1.5113003

Aberration-corrected cryogenic objective mirror with a 0.93 numerical aperture

Applied Physics Letters 115, 033701 (2019); https://doi.org/10.1063/1.5110546

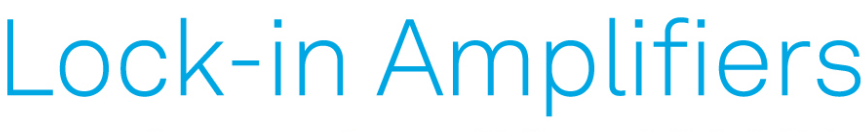

... and more, from DC to $600 \mathrm{MHz}$

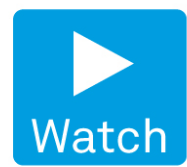




\title{
Evidence for the formation of metallic In after laser irradiation of InP
}

Cite as: J. Appl. Phys. 126, 025902 (2019); doi: $10.1063 / 1.5109230$

Submitted: 7 May 2019 . Accepted: 21 June 2019.

Published Online: 12 July 2019

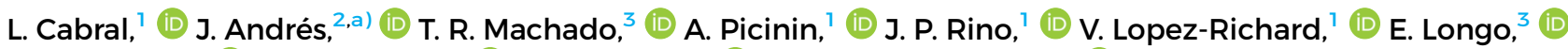
A. F. Gouveia, ${ }^{4}$ (D) G. E. Marques, ${ }^{7}$ (D) E. Z. da Silva, ${ }^{5}$ (D) and M. A. San-Miguel ${ }^{6}$ (D)

\author{
AFFILIATIONS \\ ${ }^{1}$ Department of Physics, Federal University of São Carlos, 13565-905 São Carlos, SP, Brazil \\ ${ }^{2}$ Department of Inorganic and Organic Chemistry, University Jaume I (UJI), Castelló 12071, Spain \\ ${ }^{3}$ LIEC-CDMF Department of Chemistry, Federal University of São Carlos, 13565-905 São Carlos, SP, Brazil \\ ${ }^{4}$ Department of Chemistry, State University of Piauí (UESPI), P.O. Box 381, 64002-150 Teresina, PI, Brazil \\ ${ }^{5}$ Institute of Physics "Gleb Wataghin" (IFCW), State University of Campinas, 13083-859 Campinas, SP, Brazil \\ ${ }^{6}$ Institute of Chemistry, State University of Campinas-Unicamp, 13083-970 Campinas, SP, Brazil
}

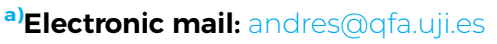

\begin{abstract}
Structural and electronic changes induced by laser irradiation are currently of interest owing to the possibility to tune the mechanical, optical, and transport properties of the irradiated materials. In this work, we investigate the effects of laser irradiation on indium phosphide, InP, by modifying the electronic temperature, $\mathrm{T}_{\mathrm{e}}$, of the system within the density functional theory framework and performing molecular dynamics simulations to prove that the laser irradiation also provokes a local thermalization effect. We found that the process can be described by a two-stage mechanism. First, at low $\mathrm{T}_{\mathrm{e}}$ values $(0-1.0 \mathrm{eV})$, the laser energy induces electronic transitions, while the InP lattice remains undisturbed and cool. In the second stage (with $\mathrm{T}_{e}$ in the range of 1.0-4.0 eV), both electron-electron scattering and electronphonon coupling processes are triggered, increasing the energy of the lattice so as to provoke a Coulomb explosion, which changes some physical chemical properties of InP. The close agreement between the simulations helps explain the formation of metallic In as it is observed in the transmission electron microscopy images.
\end{abstract}

Published under license by AIP Publishing. https://doi.org/10.1063/1.5109230

\section{INTRODUCTION}

Understanding the laser-induced stimuli at solid materials that provoke both chemical and structural perturbations is crucial to many interdisciplinary fields. In particular, laser irradiation of semiconductors to produce new materials with different functions and innovative technological advances continues to receive much attention. ${ }^{1-7}$ Although this topic attracts significant interest, as it is promising for a wide range of applications, the phenomenology of the optical heating process is complex and requires consideration of a wide set of features related to laser interaction with semiconductors to enhance the controllability of the target modification. Many theoretical and experimental studies have been devoted to this purpose. ${ }^{8-10}$ Laser irradiation is a process that is fundamentally different from chemical nanoparticle synthesis routes. In the past, different laser synthesis and processing of colloids, metal-oxides films, and nanostructures have been successfully carried out using femtosecond pulses. ${ }^{11,12}$ The strong electrical field generated by the laser light is sufficient to remove electrons from the bulk of the penetrated volume of the material. The free electrons oscillate within the electromagnetic field, transferring energy to the lattice. The irradiated surface is then heated, and this process is characterized by very fast and localized photothermal effects. The laser that focused on the surface generates a local temperature field at the desired position, allowing precise control of the local material properties.

Our research endeavors are devoted to studying the interaction between electron beams and laser irradiation with matter via the joint use of theoretical calculations and advanced experimental techniques to obtain and develop novel systems with technological applications. ${ }^{13-15}$ We have reported four new phenomena caused 
by femtosecond laser irradiation, enabling the fabrication of new complex nanoensembles, such as (i) the scale-up of the formation of $\mathrm{Ag}$ nanoparticles on $\alpha$-Ag2WO4 with bactericidal properties by femtosecond laser irradiation; ${ }^{16,17}$ (ii) the formation of nanoparticles of In from the laser irradiation of InP; ${ }^{18}$ (iii) the synthesis of stable and metastable phases of Bi nanoparticles; ${ }^{19}$ (iv) the synthesis of Ag-Bi nanoalloys with high antibacterial activity by femtosecond laser irradiations, which is interesting for potential antimicrobial applications; ${ }^{20}$ and (v) the laser and electron beam-induced formation of $\mathrm{Ag} / \mathrm{Cr}$ structures on $\mathrm{Ag}_{2} \mathrm{CrO}_{4}{ }^{21}$ In addition, we have analyzed the nature of chemical interactions by developing a new protocol based on the chemical pressure approach to point out the connection between some topological features of the electron density and structural stability of InP, thus providing an efficient tool to predict the breaking and formation of chemical bonds provoked by electron irradiation. ${ }^{22}$

The current understanding of the processes that emerge in a material upon interaction with a laser involves multiple time and length scales. ${ }^{23}$ These processes create extreme nonequilibrium conditions that provoke the loss of periodicity at the irradiated crystal, thus inducing significant perturbations on the system under observation, including chemical-bond-breaking processes, and generating transient states. These states arise through the interplay of nonequilibrium, many-body Coulomb interactions, thermal, and nonthermal effects. The excitation of electrons or collective charge oscillations and atomic ionization, with the occupation of higher energy states, eventually induces electron-electron collisions. ${ }^{24}$ In this sense, samples under laser irradiation are in a state far from equilibrium caused mainly by electronic excitations occurring in subfemtosecond time scales. Subsequently, the return to new equilibrium states involves electronic and ionic relaxations, which extend well into the picosecond range. In this process, an electronic energy redistribution takes place, and as the new lattice attains equilibrium, the additional electronic energy must be dissipated, changing the vibrational modes of the lattice. The dissipation channels for this relaxation include the electron-phonon and phonon-phonon couplings, responsible for inducing local heating of the lattice. ${ }^{25-29}$ Theoretical calculations can provide some insights into these processes via a two-temperature model, in which it is assumed that electrons are excited by the laser effect and return to an equilibrium state so rapidly that it is possible to define an electronic temperature $\left(\mathrm{T}_{\mathrm{e}}\right)$ that is much higher than the lattice temperature $\left(\mathrm{T}_{1}\right)$.

The nature of the mechanisms of the response of crystalline materials to laser irradiation remains controversial and was the subject of intense debates. ${ }^{30-32}$ The main concern of the paper was to draw attention to the nonequilibrium process of laser irradiation of matter as well as the nonequilibrium electronic excitations triggered by it. Thus, we present, for the first time, first-principles calculations based on the finite-temperature density functional theory $(\text { FT-DFT })^{33-36}$ to study electronic excitations of InP induced by femtosecond laser irradiation. The FT-DFT framework has proven its performance in understanding and rationalizing the properties of $\mathrm{Si}^{37,38} \mathrm{InSb}^{39}$ and $\mathrm{SrTiO}_{3},{ }^{24}$ semiconductors, and metals due to the presence of laser-irradiation-induced excited electrons. ${ }^{40-42}$

Indium phosphide, InP, is a III-V semiconductor with a zinc blend crystal structure and $F \overline{4} 3 \mathrm{~m}$ symmetry and has attracted considerable interest for its versatile use in electronics and optoelectronics. It has been widely explored, for instance, in applications in semiconductor devices, ${ }^{43,44}$ where the high absorption coefficient allows the InP to be engineered as efficient photodetectors, ${ }^{45}$ nanowire lasers, ${ }^{46}$ and quantum-dot light emitters. ${ }^{47,48}$ Here, the electronic excitation of InP provoked by the laser irradiation is the focus of our investigation, which is threefold. First, we are interested in exploring the efficiency of this formalism to properly describe the bond formation and rupture processes under the laser irradiation of InP. Second, we analyze and compare the modifications of the structural, electronic, and vibrational properties produced by the femtosecond laser irradiation and establish a correlation between these changes. Finally, we present the results obtained by classical molecular dynamics (MD) simulations at different temperatures (in the range of 10-1500 K) to compute the phonon dispersion and specific heat in order to prove that the laser irradiation also provokes a local thermalization effect. With these results, we wish to answer the following questions: (1) how the laser irradiation changes structural properties, such as lattice parameter, In-P bond distances, and elastic constants along with electronic properties such as band structure, electron density distributions, and dielectric constants; (2) how the phonon vibrational modes are modified by laser irradiation, showing that the twotemperature theoretical framework yields similar effects associated to lattice temperature changes as the classical MD simulations; and (3) how the laser effects allow forming metallic In, as observed in our reported experimental results.

\section{THEORETICAL APPROACH AND COMPUTATIONAL DETAILS}

The Perdew-Burke-Ernzerhof (PBE) functional ${ }^{49}$ was selected for our calculations. This functional systematically underestimates the bandgap energy of semiconductor materials, and hybrid functionals such as HSE06 and PBE0, ${ }^{50-52}$ the DFT+U method, ${ }^{53}$ or other many-body perturbation methods should be used for improving the gap description. However, their use involves a much higher computational cost. In this work, we focus on the effects of the electronic temperature, and although the exact energy values of the electronic excitations are modified by the band gap underestimation, the trend of the electronic properties should not be affected.

The Kohn-Sham equations were solved using the projected augmented-wave (PAW) method, ${ }^{54,55}$ as implemented in the Vienna $a b$ initio simulation package (VASP), ${ }^{56,57}$ version 5.4.4, employing the following PAW projectors: $\operatorname{In}\left(4 \mathrm{~d}^{10} 5 \mathrm{~s}^{2} 5 \mathrm{p}^{1}\right)$ and $\mathrm{P}\left(3 \mathrm{~s}^{2} 3 \mathrm{p}^{3}\right)$, where the valence electrons are shown in parenthesis. The equilibrium of the InP bulk was reached by a minimization of the stress tensor (atomic forces) using a plane wave cutoff of $557 \mathrm{eV}(313 \mathrm{eV})$, where the cutoff employed for atomic forces is $12.5 \%$ higher than the largest recommended PAW cutoff energy for the mentioned chemical species. Here, the difference between the cutoff energies is due to the slow (fast) convergence of stress tensor (total energy) as a function of the number of used plane waves. The integration of the Brillouin zone was performed employing a k-mesh of $5 \times 5 \times 5$ for the InP bulk optimization and twice for electronic properties. For these calculations, we used a selfconsistent electronic convergence criterium of $10^{-6} \mathrm{eV}$, where the 
equilibrium geometries were reached when the atomic forces on every atom were smaller than $0.01 \mathrm{eV} \AA^{-1}$.

To better understand the effects of the electronic excitation in the InP and its electronic occupations $\rho_{i}$, discretized in energy levels $\varepsilon_{j}$, we considered that fermionic excitations to the conduction band $(\mathrm{CB})$ are only due to the increase in the electronic temperature $\mathrm{T}_{\mathrm{e}}$, where the electronic occupation obey the Fermi-Dirac statistics within the DFT scheme $\rho_{i}=\left[\exp \left(\frac{\varepsilon_{j}-\mu}{\sigma}+1\right)\right]^{-1},{ }^{58}$ where $\sigma=\mathrm{K}_{\mathrm{B}} \mathrm{T}_{\mathrm{e}}, \mathrm{K}_{\mathrm{B}}$ is the Boltzmann constant and $\mu$ is the chemical potential provided by the atomic species in the periodic calculation. The convenient variation of the electronic temperature induces electron density rearrangements in the system, and then new atomic positions can be obtained.

The electron density redistributions and charge transfer processes as a function of $\mathrm{T}_{e}$ are investigated by using the quantum theory of atoms in molecules (QTAIM) ${ }^{59,60}$ and the electron localization function (ELF) ${ }^{61-63}$ The calculation of the vibrational lattice modes was performed through the phonon frequencies. ${ }^{64-67}$ The force constants were calculated within the frozen phonons approach as implemented in the Phonopy package, ${ }^{68,69}$ where the k-mesh used was $10 \times 10 \times 10$. The vibrational frequencies were obtained within the harmonic approximation by calculating the numerical second derivatives of the potential energy using the scheme implemented in the Phonopy package.

The stress tensor was also investigated within the scope of Hook's law. ${ }^{70}$ In the particular case of InP, with a zinc blend bulk phase, the calculation of the elastic properties is reduced to only three nonequivalent elastic constants $\mathrm{C}_{11}, \mathrm{C}_{44}$, and $\mathrm{C}_{12}$. Then, the bulk modulus can be obtained as $\mathrm{B}_{0}=1 / 3\left(\mathrm{C}_{11}+2 \mathrm{C}_{12}\right)$ and the shear modulus as $S_{0}=1 / 2\left(C_{11}-C_{12}\right){ }^{71,72}$ These calculations allow us to assess the effect of strain and the change of the specific heat induced by the laser irradiation. In order to obtain a better description of the elastic constants for the PBE functional, we employed the cutoff energy multiplied by a factor 2.5, namely, $696 \mathrm{eV}$. For those calculations, the finite differences allowed the calculation of the Hessian matrix, where we used the atomic step size of $0.010 \AA$, which is smaller than the one recommended by VASP package. Molecular dynamics simulations were also performed based on the effective interaction potential proposed by Branicio et al. ${ }^{73}$ A system with 64000 particles was simulated in the NPT ensemble (constant number of particles, pressure, and temperature) for temperatures ranging from 10 to $1500 \mathrm{~K}$. The phonon vibrational density of states (VDOS) was obtained by the Fourier Transform of the velocity autocorrelation function as a function of temperature. The softening due to the increase in temperature of the VDOS was then compared with the results obtained from DFT calculations. More details can be found in the supplementary material.

\section{RESULTS AND DISCUSSION}

To understand the effects of the laser irradiation on the pristine InP crystal, first, analysis of the variation in the lattice parameter, In-P bond, bulk modulus, and shear modulus is presented. Second, we will analyze the behavior of the electronic properties by calculating the charge redistribution of In and $\mathrm{P}$ atoms, electronic density of states, dielectric function, and the electron localization function (ELF). In addition, the band structure and the electronic occupations as a function of the electronic temperature were analyzed, providing an elucidative study of the electronic levels' positions. A comprehensive study of the vibrational modes and structure stability through the phonons is presented as well. Finally, molecular dynamics simulations have been performed to elucidate the thermal effects associated with the laser irradiation.

\section{A. Structural properties}

The laser irradiation provides high energy doses to the material conducting eventually to significant structural distortions and formation of defects. We initiate these studies by searching the optimal InP structure as $\mathrm{T}_{e}$ increases. The evolution of the optimized lattice constant $\mathrm{a}_{0}$ and In-P distance is shown in Fig. 1. Analysis of these results shows that, for small electronic temperatures, the equilibrium lattice constant is $\mathrm{a}_{0}=5.96 \AA$, which agrees with the experimental value of $\mathrm{a}_{0}=5.87 \AA$. The value of $\mathrm{a}_{0}$, as well as for the In-P distances, shows a smooth increase as $\mathrm{T}_{\mathrm{e}}$ grows. In particular, the effects of the electronic temperature ranging $0-1.5 \mathrm{eV}$ on the InP distances and lattice parameters are negligible compared to $\mathrm{T}_{\mathrm{e}}$ higher than $1.5 \mathrm{eV}$.

A deeper understanding of the effects of the laser irradiation on the geometry can be elucidated by calculating the elastic constants, bulk, and shear modulus, as presented in Fig. 2. For small electronic temperature calculations, such as $\mathrm{T}_{\mathrm{e}}=0-0.1 \mathrm{eV}$, the obtained theoretical predictions (experimental measurements, reported in Refs. 74 and 75$)$, are $\mathrm{C}_{11}=87.48 \mathrm{GPa}\left(\mathrm{C}_{11}=101.00\right.$ $\mathrm{GPa}), \mathrm{C}_{12}=46.11 \mathrm{GPa}\left(\mathrm{C}_{11}=56.00 \mathrm{GPa}\right)$, and $\mathrm{C}_{44}=41.60 \mathrm{GPa}$ $\left(\mathrm{C}_{44}=46.00 \mathrm{GPa}\right)$. This disagreement can be attributed to the failures of the PBE functional. An analysis of the results indicates that, for lower $\mathrm{T}_{\mathrm{e}}=0-0.01 \mathrm{eV}$, small perturbations in the values of the elastic constants are sensed. By increasing the electronic temperature, we note a strong decrease in the elastic constants, inducing changes on the internal strains. Thus, the three Born stability criteria for the cubic system begin to not be met: $\mathrm{C}_{11}-\mathrm{C}_{12}>0$; $\mathrm{C}_{11}+2 \mathrm{C}_{12}>0 ; \mathrm{C}_{44}>0 .^{76,77}$ This behavior indicates that the atoms are moving away from the ideal positions, increasing the interatomic distances, which is in agreement with the results presented in Fig. 2.

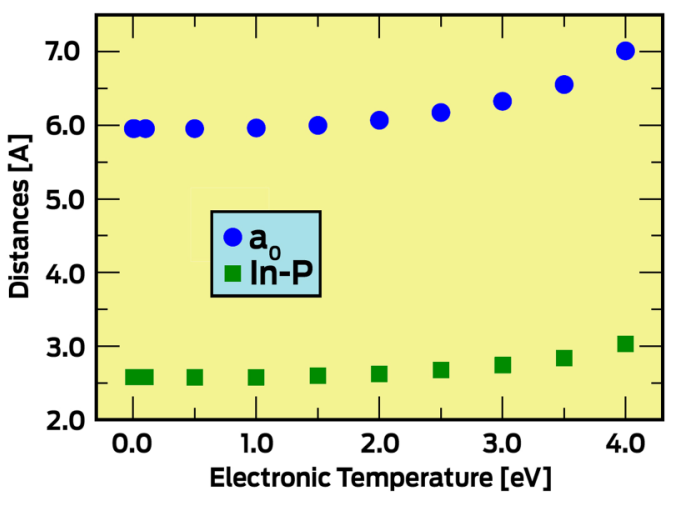

FIG. 1. Values of the calculated lattice parameter $a_{0}$ and the In-P distance as a function of the electronic temperature, $\mathrm{T}_{\mathrm{e}}$. 

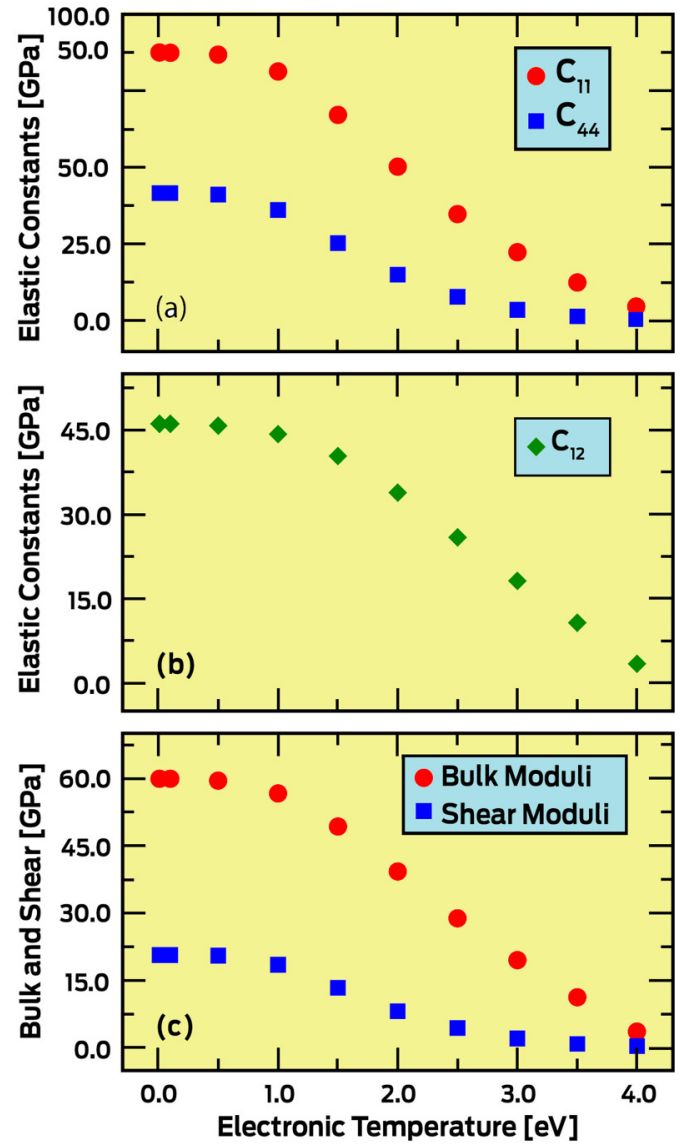

FIG. 2. Calculated elastic constants (a) $\mathrm{C}_{11}$ and $\mathrm{C}_{44}$, (b) $\mathrm{C}_{12}$, and bulk and shear modulus (c) as a function of $\mathrm{T}_{\mathrm{e}}$.

\section{B. Electronic properties}

InP is a nonmagnetic semiconductor, with a direct gap in the $\Gamma$-point. At $\mathrm{T}_{\mathrm{e}}$ within the range of $0-1.0 \mathrm{eV}$, we note that the valence band $(\mathrm{VB})$ maximum is triply degenerate, in contrast with the nondegenerate empty state in the conduction band (CB) minimum. We also find that the occupancy of the energy levels and Fermi energy for the interval between $T_{e}=0-0.1 \mathrm{eV}$ does not present significant changes except for a minor variation on the positions of some energy levels (see the band structure calculation in the supplementary material). By increasing $\mathrm{T}_{\mathrm{e}}$, the lowest levels of the $\mathrm{CB}$ band gradually becomes occupied, where the $\mathrm{CB}$ minimum is the preferential state for the occupation of the excited electrons. For $\mathrm{T}_{\mathrm{e}}=0.5 \mathrm{eV}$, the Fermi energy is higher than the $\mathrm{VB}$ maximum energy for small electronic temperatures. Moreover, for higher electronic temperatures, such as $\mathrm{T}_{\mathrm{e}}=2.5 \mathrm{eV}$, the $\mathrm{VB}$ maximum and $\mathrm{CB}$ minimum are triply degenerate, where $\mathrm{CB}$ is partially filled. Furthermore, a thorough analysis of energy levels indicated that the excited electrons in the $\mathrm{CB}$ originated from the higher levels of the $\mathrm{VB}$ as $\mathrm{T}_{\mathrm{e}}$ increases, and, then, more electrons are located in the $\mathrm{CB}$ and can be excited from inner levels in the VB. The electronic density of states projected on the different atomic orbitals ( $\mathrm{s}, \mathrm{p}$, and $\mathrm{d}$ ) at $\mathrm{T}_{\mathrm{e}}=0.01 \mathrm{eV}$ (see the supplementary material) shows that both $\mathrm{p}$ states of In and $\mathrm{P}$ atoms are the main components of the Fermi level and have the larger contribution to the VB.

The increase of $T_{e}$ provides changes in the energy levels positions and, as a consequence, new electronic transitions can be triggered. Thus, the electronic charge transfer occurs from $\mathrm{P}$ to In atoms, and an analysis of the corresponding orbitals indicates that this electronic flow takes place between the $\mathrm{P}$ and In p-orbitals. In order to elucidate this process, we irradiated an InP sample using a femtosecond laser and compared with the pristine material. We observed the formation of metallic In, proving the charge transfer between atoms and providing the ripple of the material until metallic In atoms are placed at the edge of the sample, as depicted in Fig. 3. In addition, the atomic displacements from equilibrium positions lead to new chemical environments involving electron density rearrangements in the system. Given the fact that $\mathrm{P}$ atoms have higher electronegativity than In atoms, electron density tends to accumulate near the $\mathrm{P}$ atoms providing an anionic character. This fact can be visualized in the ELF map reported in the supplementary material.

The effect of $T_{e}$ changes on the electron density distribution was evaluated by calculating the electronic charge enclosed within the Bader atomic basins for the two (In and P) atom types. The effective Bader charge $\left(Q_{e f f}^{B}\right)$ is calculated as $Q_{\text {eff }}^{B}=Z_{v a l}-Q^{\text {Bader }}$, where the $Z_{v a l}$ are the number of valence electrons and $Q^{\text {Bader }}$ is the

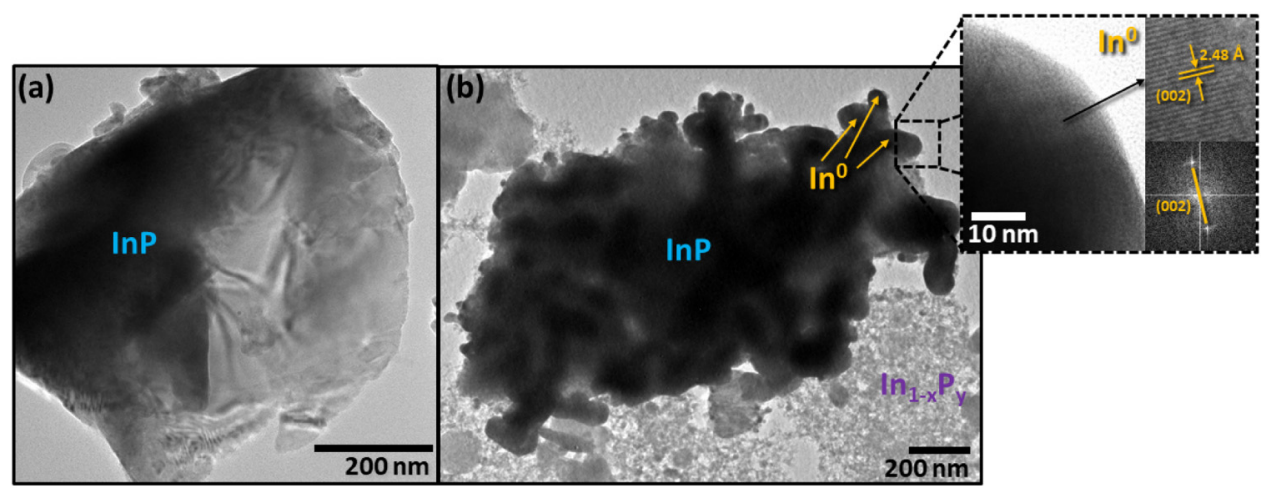

FIG. 3. Transmission electron microscopy (TEM) images. The InP pristine material is shown (a) before the laser irradiation and (b) after electronic excitation by laser action, where the presence of $\ln$ is obtained. 


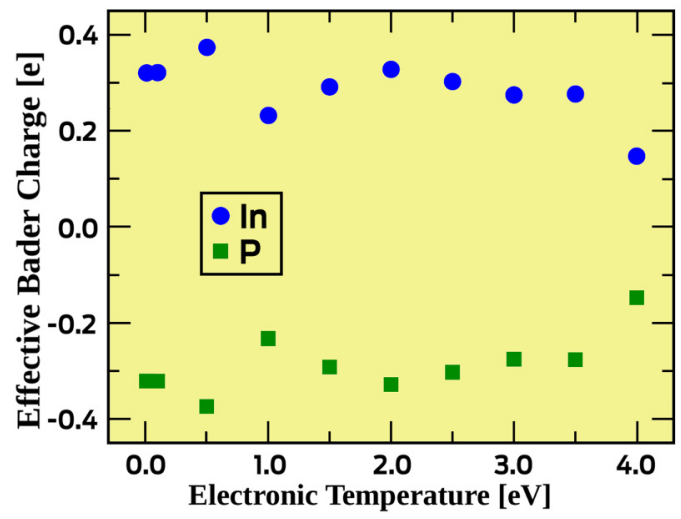

FIG. 4. Calculated values of the effective Bader charges as a function of $T_{e}$.

Bader charge. The calculated effective Bader charges for both atoms are represented in Fig. 4. The results show how the increase of $\mathrm{T}_{\mathrm{e}}$ affects the atomic charge of both atoms. In fact, as rising $\mathrm{T}_{\mathrm{e}}$, significant changes in the effective Bader charges are observed. In the range $0-1.0 \mathrm{eV}$ for $\mathrm{T}_{\mathrm{e}}$, the atomic charges oscillate between $0.37 e$ and $0.23 e(-0.37 e$ and $-0.23 e)$ for In $(\mathrm{P})$ atoms, whereas in the range of $\mathrm{T}_{\mathrm{e}}, 1.0-4.0 \mathrm{eV}$, these values oscillate between $0.33 e$ and $0.15 e(-0.37 \mathrm{e}$ and $-0.23 e)$. We clearly note a tendency of the In atoms to assume the metallic character, similarly to that effect observed when the material is highly dosed with electrons irradiated by transmission electron microscopy (TEM) beams. ${ }^{22}$ We have also investigated the laser effects on the dielectric function at different electronic temperatures for InP (see the supplementary material). The most significant differences are observed at the highest temperatures when the material experience profound transformations as discussed previously and the optical properties also reflect that fact.

\section{Phonon vibrational frequencies and lattice temperature effects}

To characterize the lattice vibrations induced by the laser incidence, we compare DFT calculations and classical molecular dynamics simulations in order to study the phonons and the specific heat of the material. First of all, we analyze the phonon frequencies at different electronic temperatures. Figure 5(a) shows the phonon dispersion of InP calculated at normal conditions (low electronic temperatures), which is in agreement with the experimental measurements. ${ }^{78-80}$ As already reported in the literature, the optical branches are quite flat because of the large mass difference between In and $\mathrm{P}$ atoms. This fact also results in the observation that acoustical and optical phonons are separated by a gap of about $5 \mathrm{THz}$. Moreover, the transverse optical (TO) and longitudinal optical (LO) modes at the $\Gamma$-point were chosen and compared with experimental and other theoretical values from the literature at normal conditions (see the supplementary material). Our estimated values are well below the experimental values, because it is known that the PBE functional underestimates drastically the

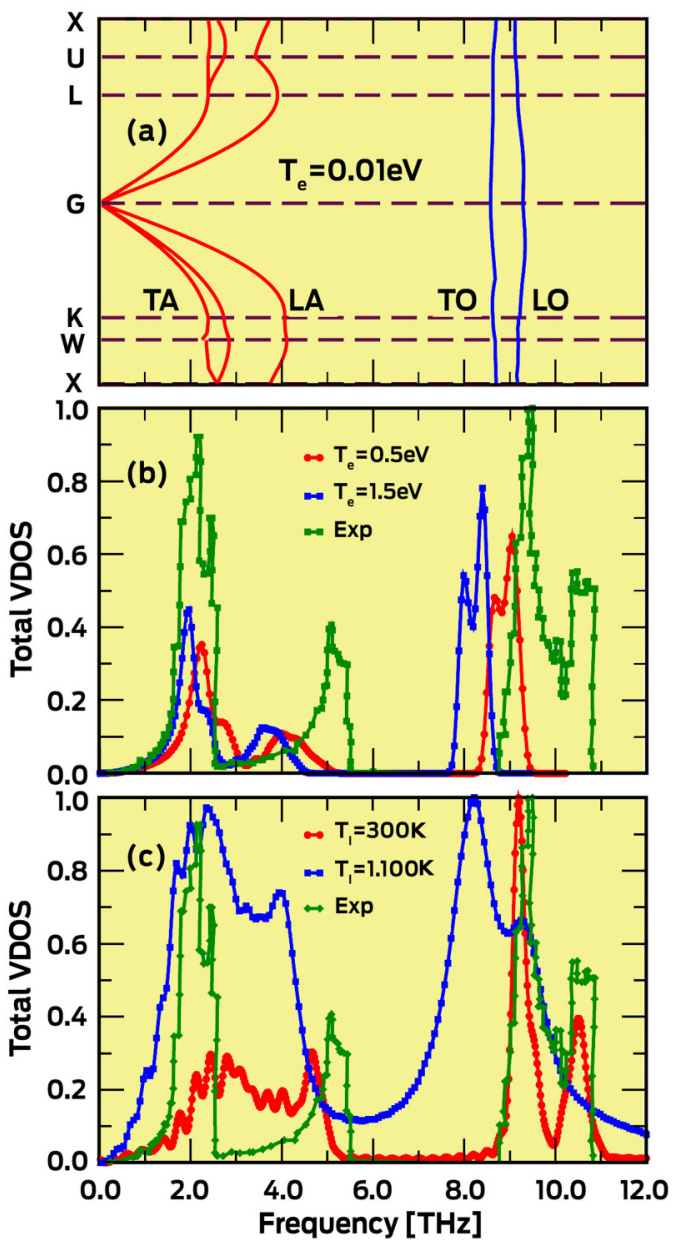

FIG. 5. (a) InP phonon band structure. The transverse acoustic (TA) and longitudinal acoustic (LA) modes are represented in red, and the transverse optical (TO) and longitudinal optical (LO) frequencies are represented in blue. Vibrational density of states (VDOS) as a function of frequency (in $\mathrm{THz}$ ) at different values of (b) $T_{e}$ and (c) $T_{1}$.

phonon frequencies. However, our aim here is to analyze the effect of the electronic excitations on the phonon frequencies to identify structural modifications due to the laser effect. It can also be seen that the vibrational density of states (VDOS) distribution is shifted when compared to the low $\mathrm{T}_{\mathrm{e}}$ regime [Fig. 5(b)]. This behavior is a consequence of the local lattice heating due to the laser irradiation. Thus, as $T_{e}$ increases, the electronic excitations rise the electron repulsions forcing atoms to move away from their original positions, as depicted also by the InP distances in Fig. 1, and consequently leading to lattice heating. Additionally, the effects of the lattice heating on the VDOS have been simulated using classical MD simulations and represented in Fig. 5(c). Thus, at $300 \mathrm{~K}$, the VDOS corresponding to the optical modes (higher frequencies) is quite similar to the experimental one, indicating that the vibrational structure of 
the InP is well represented by the force field. When the system is heated to $1500 \mathrm{~K}$, the phonon softening is observed and the distribution is shifted to lower frequencies. In addition, the equivalence between the DFT and MD simulations is provided in the supplementary material as a function of the electronic and lattice temperatures for the InP lattice parameter.

We also calculated the $\mathrm{LO}$ and $\mathrm{TO}$ vibrational phonon modes at the $\Gamma$-point as a function of $\mathrm{T}_{\mathrm{e}}$ by using the DFT PBE exchange correlation functional, depicted in Fig. 6(a), where the increase in the electronic temperature decreases the LO and TO vibrational InP frequencies. In fact, it can be seen that at low electronic temperatures (lower than $1 \mathrm{eV}$ ), there are negligible changes for the optical frequencies, compatible with a regime of lattice stability. For $\mathrm{T}_{\mathrm{e}}>1.0 \mathrm{eV}$, the structural modifications become stronger, which was previously elucidated, and the elastic constants calculations are depicted in Fig. 2.

Furthermore, as the electronic excitation can contribute to the heat transfer and thermal expansion of the material, we compared the specific heat of InP calculated from integrating the DFT phonon density of states for $\mathrm{T}_{\mathrm{e}}=0.01 \mathrm{eV}$ and classical MD simulations. These results are plotted in Fig. 6(b) and show that both methodologies are in good agreement to represent the effect of the lattice heating on the thermal properties.
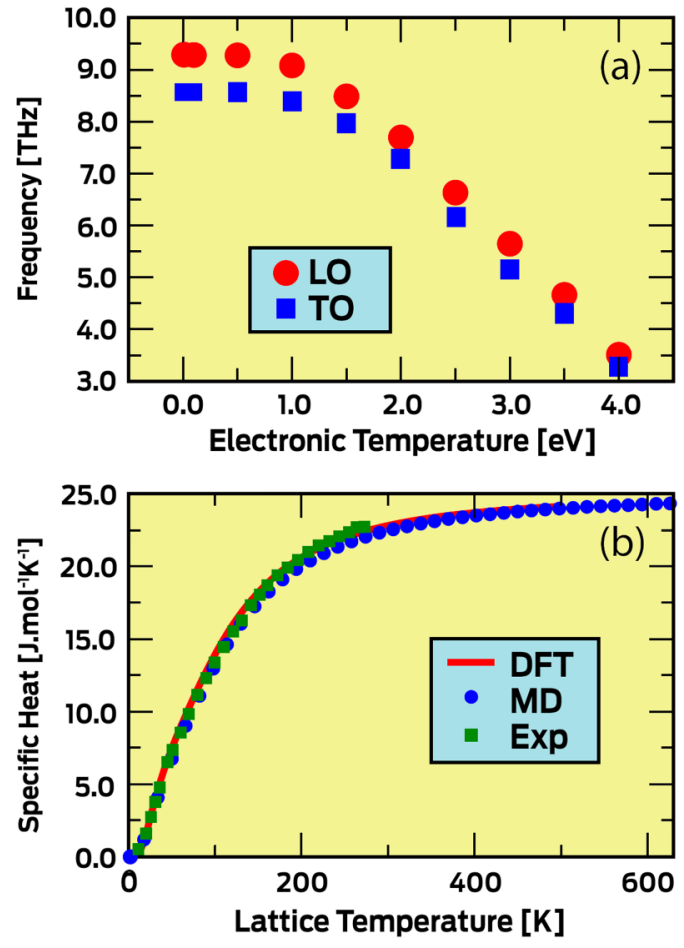

FIG. 6. (a) LO and TO vibrational phonon modes, calculated at the DFT level as a function of $T_{e}$. (b) Values of the specific heat as a function of the lattice temperature obtained by using DFT calculations and MD simulations. Experimental values are included for comparison.

\section{CONCLUSIONS}

With external perturbations, such as laser irradiation, the nature of a material changes from its ground state and exhibits interesting properties. However, the response of solids to highpower laser irradiation is not well understood. This work sought some understanding of the effects on InP induced by laser irradiation. The laser excitations were simulated by performing density functional theory calculations within the electronic temperature approach, which allows us to analyze the structural and electronic properties at the atomic level.

The laser irradiation of InP crystals was selected as an example. Increasing the value of $\mathrm{T}_{e}$ in the bulk structure results in an increase in both the lattice parameter, and In-P distance, which changes the chemical environment of the InP bulk phase. The direct consequence of the lattice parameter increase is the decrease in the elastic constants, bulk, and shear modulus of the material. The Bader charge analysis shows that the In atoms attract more electrons than $\mathrm{P}$ atoms to fill their empty subshells and become neutral at larger values of $\mathrm{T}_{\mathrm{e}}$. In fact, this process induces changes in the ELF around In atoms and along In-P contacts. We found that small values of $\mathrm{T}_{\mathrm{e}}$ ensure that states in the valence band are occupied and the forbidden region of the semiconductor is well defined.

An increment of $T_{e}$ values enhances the electron excitation process from the valence to the conduction bands. Larger values of electronic temperatures change the symmetry of the energy levels, which are triply degenerate in the valence band for small values of $\mathrm{T}_{\mathrm{e}}$. Another consequence of the electron excitations is the significative changes in the dielectric function of the material, thereby changing the optical response of the irradiated material. These structural and electronic modifications contribute to new vibrational phonon mode frequencies, thus affecting the heat capacity of the material.

A comparison of the results obtained by the density functional theory and molecular dynamics simulations shows good agreement, pointing out that the laser irradiation provokes a local thermalization effect. The present results are capable to explain the formation of metallic In when InP is irradiated by laser as it is observed in the transmission electron microscopy images.

\section{SUPPLEMENTARY MATERIAL}

See the supplementary material for structural properties; dielectric function, density of states, and band structure; electron localization function-ELF; electronic and lattice temperature-lattice parameter; electronic temperature and vibrational phonon modes; and experimental approach.

\section{ACKNOWLEDGMENTS}

The authors thank CAPES (Coordenação de Aperfeiçoamento de Pessoal de Nível Superior)—finance code 1-PNPD program, CNPq (Conselho Nacional de Desenvolvimento Científico e Tecnológico) (Grant No. 150949/2018-9), and FAPESP (Fundação de Amparo à Pesquisa do Estado de São Paulo) (Grant Nos. 2014/ 19142-2, 2013/07296-2, and 2016/23891-6) for financial support from Brazil. This work used computational resources of the 
"Centro Nacional de Processamento de Alto Desempenho em São Paulo" (CENAPAD-SP), "Centro de Computação John David Rogers" (CCJDR-UNICAMP), and the CENAPAD-RJ (SDumont). The authors thank Enio Longo for design contributions. J.A. acknowledges Generalitat Valenciana for PrometeoII/2014/022, ACOMP/2015/1202, Ministerio de Economia y Competitividad, project CTQ2015-65207-P, and Universitat Jaume I, Spain (Project No. UJI-B2016-25). The authors are very grateful to Professor Dr. Gladys Mínguez Vega, Professor Dr. Eloisa Cordoncillo, Professor Dr. Héctor Beltran Mir, Verónica Puerto-Belda, and the "Servei Central d'Instrumentació Científica (SCIC)" of the University Jaume I for the use of the femtosecond laser equipment and scientific discussion of obtained results.

\section{REFERENCES}

${ }^{1}$ R. Buividas, M. Mikutis, and S. Juodkazis, "Surface and bulk structuring of materials by ripples with long and short laser pulses: Recent advances," Prog. Quant. Electron. 38, 119-156 (2014).

${ }^{2}$ W. Xiong, Y. Zhou, W. Hou, L. Jiang, M. Mahjouri-Samani, J. Park, X. He, Y. Gao, L. Fan, T. Baldacchini, J.-F. Silvain, and Y. Lu, "Laser-based micro/nanofabrication in one, two and three dimensions," Front. Optoelectron. 8, 351-378 (2015).

${ }^{3}$ M. Malinauskas, A. Žukauskas, S. Hasegawa, Y. Hayasaki, V. Mizeikis, R. Buividas, and S. Juodkazis, "Ultrafast laser processing of materials: From science to industry," Light Sci. Appl. 5, el6133 (2016).

${ }^{4}$ S. Jesse, A. Y. Borisevich, J. D. Fowlkes, A. R. Lupini, P. D. Rack, R. R. Unocic, B. G. Sumpter, S. V. Kalinin, A. Belianinov, and O. S. Ovchinnikova, "Directing matter: Toward atomic-scale 3D nanofabrication," ACS Nano 10, 5600-5618 (2016).

${ }^{5}$ D. Tan, K. N. Sharafudeen, Y. Yue, and J. Qiu, "Femtosecond laser induced phenomena in transparent solid materials: Fundamentals and applications," Prog. Mater. Sci. 76, 154-228 (2016).

${ }^{6}$ T. Satoh, R. Iida, T. Higuchi, Y. Fujii, A. Koreeda, H. Ueda, T. Shimura, K. Kuroda, V. I. Butrim, and B. A.Ivanov, "Excitation of coupled spin-orbit dynamics in cobalt oxide by femtosecond laser pulses," Nat. Commun. 8, 638 (2017).

${ }^{7}$ L. Jiang, A.-D. Wang, B. Li, T.-H. Cui, and Y.-F. Lu, "Electrons dynamics control by shaping femtosecond laser pulses in micro/nanofabrication: Modeling, method, measurement and application," Light Sci. Appl. 7, 17134 (2018).

${ }^{8}$ T. J.-Y. Derrien, T. E. Itina, R. Torres, T. Sarnet, and M. Sentis, "Possible surface plasmon polariton excitation under femtosecond laser irradiation of silicon,” J. Appl. Phys. 114, 083104 (2013).

${ }^{9}$ J. Z. P. Skolski, G. R. B. E. Römer, J. V. Obona, V. Ocelik, A. J. Huis in’t Veld, and J. T. M. De Hosson, "Laser-induced periodic surface structures: Fingerprints of light localization,” Phys. Rev. B 85, 075320 (2012).

${ }^{10}$ J. Bonse, J. Krüger, S. Höhm, and A. Rosenfeld, "Femtosecond laser-induced periodic surface structures,” J. Laser Appl. 24, 042006 (2012).

${ }^{11}$ D. Zhang, B. Gökce, and S. Barcikowski, "Laser synthesis and processing of colloids: Fundamentals and applications,” Chem. Rev. 117, 3990-4103 (2017).

${ }^{12}$ H. Palneedi, J. H. Park, D. Maurya, M. Peddigari, G.-T. Hwang, V. Annapureddy, J.-W. Kim, J.-J. Choi, B.-D. Hahn, S. Priya, K. J. Lee, and J. Ryu, "Laser irradiation of metal oxide films and nanostructures: Applications and advances," Adv. Mater. 30, 1705148 (2018).

${ }^{13}$ M. A. San-Miguel, E. Z. da Silva, S. M. Zanetti, M. Cilense, M. T. Fabbro, L. Gracia, J. Andrés, and E. Longo, "In situ growth of Ag nanoparticles on $\alpha-\mathrm{Ag}_{2} \mathrm{WO}_{4}$ under electron irradiation: Probing the physical principles," Nanotechnology 27, 225703 (2016).

${ }^{14}$ R. C. de Oliveira, M. Assis, M. M. Teixeira, M. D. P. da Silva, M. S. Li, J. Andres, L. Gracia, and E. Longo, "An experimental and computational study of $\beta-\mathrm{AgVO}_{3}$ : Optical properties and formation of $\mathrm{Ag}$ nanoparticles," J. Phys. Chem. C 120, 12254-12264 (2016).

${ }^{15}$ J. Andrés, A. F. Gouveia, L. Gracia, E. Longo, F. G. Manzeppi, E. Z. da Silva, D. H. Pereira, and M. A. San-Miguel, "Formation of Ag nanoparticles under electron beam irradiation: Atomistic origins from first-principles calculations," Int. J. Quantum Chem. 118, e25551 (2017).

${ }^{16} \mathrm{M}$. Assis, E. Cordoncillo, R. Torres-Mendieta, H. Beltrán-Mir, G. Mínguez-Vega, R. Oliveira, E. R. Leite, C. C. Foggi, C. E. Vergani, E. Longo, and J. Andrés, "Towards the scale-up of the formation of nanoparticles on $\alpha-\mathrm{Ag}_{2} \mathrm{WO}_{4}$ with bactericidal properties by femtosecond laser irradiation," Sci. Rep. 8, 1884 (2018).

${ }^{17}$ N. G. Macedo, T. R. Machado, R. A. Roca, M. Assis, C. C. Foggi, V. Puerto-Belda, G. Mínguez-Vega, A. Rodrigues, M. A. San-Miguel, E. Cordoncillo, H. Beltrán-Mir, J. Andrés, and E. Longo, "Tailoring the bactericidal activity of $\mathrm{Ag}$ nanoparticles $/ \alpha-\mathrm{Ag}_{2} \mathrm{WO}_{4}$ composite induced by electron beam and femtosecond laser irradiation: Integration of experiment and computational modeling," ACS Appl. Bio Mater. 2, 824-837 (2019).

${ }^{18}$ M. Assis, N. G. Macedo, T. R. Machado, M. M. Ferrer, A. F. Gouveia, E. Cordoncillo, R. Torres-Mendieta, H. Beltrán-Mir, G. Mínguez-Vega, E. R. Leite, J. R. Sambrano, J. Andrés, and E. Longo, "Laser/electron irradiation on indium phosphide (InP) semiconductor: Promising pathways to in situ formation of indium nanoparticles," Part. Part. Syst. Char. 35, 1800237 (2018).

${ }^{19} \mathrm{M}$. Assis, E. Cordoncillo, R. Torres-Mendieta, H. Beltán-Mir, G. Mínguez-Vega, A. F. Gouveia, E. Leite, J. Andrés, and E. Longo, "Laser-induced formation of bismuth nanoparticles," Phys. Chem. Chem. Phys. 20, 13693-13696 (2018).

${ }^{20}$ T. R. Machado, N. G. Macedo, M. Assis, C. Doñate Buendia, G. Mínguez-Vega, M. M. Teixeira, C. C. Foggi, C. E. Vergani, H. Beltrán-Mir, J. Andrés, E. Cordoncillo, and E. Longo, "From complex inorganic oxides to Ag-Bi nanoalloy: Synthesis by femtosecond laser irradiation,” ACS Omega 3, 9880-9887 (2018).

${ }^{21}$ P. S. Lemos, G. S. Silva, R. A. Roca, M. Assis, R. Torres-Mendieta, H. Beltrán-Mir, G. Mínguez-Vega, E. Cordoncillo, J. Andrés, and E. Longo, "Laser and electron beam-induced formation of $\mathrm{Ag} / \mathrm{Cr}$ structures on $\mathrm{Ag}_{2} \mathrm{CrO}_{4}$," Phys. Chem. Chem. Phys. 21, 6101-6111 (2019).

${ }^{22}$ H. H. Osman, J. Andrés, M. A. Salvadó, and J. M. Recio, "Chemical bond formation and rupture processes: An application of DFT-chemical pressure approach," J. Phys. Chem. C 122, 21216-21225 (2018).

${ }^{23}$ K. K. Ostrikov, F. Beg, and A. Ng, "Colloquium: Nanoplasmas generated by intense radiation," Rev. Mod. Phys. 88, 011001 (2016).

${ }^{\mathbf{2 4}} \mathrm{S}$. Zhao, Y. Zhang, and W. J. Weber, "Ab initio study of electronic excitation effects on $\mathrm{SrTiO}_{3}$," J. Phys. Chem. C 121, 26622-26628 (2017).

${ }^{25}$ S. Gerber, S.-L. Yang, D. Zhu, H. Soifer, J. A. Sobota, S. Rebec, J. J. Lee, T. Jia, B. Moritz, C. Jia, A. Gauthier, Y. Li, D. Leuenberger, Y. Zhang, L. Chaix, W. Li, H. Jang, J.-S. Lee, M. Yi, G. L. Dakovski, S. Song, J. M. Glownia, S. Nelson, K. W. Kim, Y.-D. Chuang, Z. Hussain, R. G. Moore, T. P. Devereaux, W.-S. Lee, P. S. Kirchmann, and Z.-X. Shen, "Femtosecond electron-phonon lock-in by photoemission and $\mathrm{x}$-ray free-electron laser," Science 357, 71-75 (2017).

${ }^{26} \mathrm{~N}$. Brouwer and B. Rethfeld, "Transient electron excitation and nonthermal electron-phonon coupling in dielectrics irradiated by ultrashort laser pulses," Phys. Rev. B 95, 245139 (2017).

${ }^{27}$ B. Y. Mueller and B. Rethfeld, "Relaxation dynamics in laser-excited metals under nonequilibrium conditions,” Phys. Rev. B 87, 035139 (2013).

${ }^{28}$ W. S. Fann, R. Storz, H. W. K. Tom, and J. Bokor, "Direct measurement of nonequilibrium electron-energy distributions in subpicosecond laser-heated gold films," Phys. Rev. Lett. 68, 2834-2837 (1992).

${ }^{29}$ X. Y. Wang, D. M. Riffe, Y.-S. Lee, and M. C. Downer, "Time-resolved electron-temperature measurement in a highly excited gold target using femtosecond thermionic emission," Phys. Rev. B 50, 8016-8019 (1994).

${ }^{30}$ C. Lian, S. B. Zhang, and S. Meng, "Ab initio evidence for nonthermal characteristics in ultrafast laser melting,” Phys. Rev. B 94, 184310 (2016). 
${ }^{31}$ T. Zier, M. E. Garcia, and P. L. Silvestrelli, "Comment on "Ab initio evidence for nonthermal characteristics in ultrafast laser melting", "Phys. Rev. B 99, 066301 (2019).

${ }^{32}$ C. Lian, S. B. Zhang, and S. Meng, "Reply to "Comment on "Ab initio evidence for nonthermal characteristics in ultrafast laser melting"'," Phys. Rev. B 99, 066302 (2019).

${ }^{33}$ N. D. Mermin, "Thermal properties of the inhomogeneous electron gas," Phys. Rev. 137, A1441-A1443 (1965).

${ }^{34}$ G. Kresse and J. Hafner, "Ab initio molecular dynamics for liquid metals," Phys. Rev. B 47, 558-561 (1993).

${ }^{35}$ A. Alavi, J. Kohanoff, M. Parrinello, and D. Frenkel, "Ab initio molecular dynamics with excited electrons,” Phys. Rev. Lett. 73, 2599-2602 (1994).

${ }^{36}$ N. Marzari, D. Vanderbilt, and M. C. Payne, "Ensemble density-functional theory for ab initio molecular dynamics of metals and finite-temperature insulators," Phys. Rev. Lett. 79, 1337-1340 (1997).

${ }^{37}$ P. L. Silvestrelli, A. Alavi, M. Parrinello, and D. Frenkel, "Structural, dynamical, electronic, and bonding properties of laser-heated silicon: An ab initio molecular-dynamics study," Phys. Rev. B 56, 3806-3812 (1997).

${ }^{38}$ P. L. Silvestrelli, A. Alavi, M. Parrinello, and D. Frenkel, "Ab initio molecular dynamics simulation of laser melting of silicon,” Phys. Rev. Lett. 77, 3149-3152 (1996).

${ }^{39}$ E. S. Zijlstra, J. Walkenhorst, and M. E. Garcia, "Anharmonic noninertial lattice dynamics during ultrafast nonthermal melting of InSb," Phys. Rev. Lett. 101, 135701 (2008).

${ }^{40}$ N. S. Grigoryan, T. Zier, M. E. Garcia, and E. S. Zijlstra, "Ultrafast structural phenomena: Theory of phonon frequency changes and simulations with code for highly excited valence electron systems," J. Opt. Soc. Am. B 31, C22-C27 (2014).

${ }^{41}$ S. Ghosh, P. Verma, C. J. Cramer, L. Gagliardi, and D. G. Truhlar, "Combining wave function methods with density functional theory for excited states," Chem. Rev. 118, 7249-7292 (2018).

${ }^{42}$ E. S. Zijlstra, F. CheenicodeKabeer, B. Bauerhenne, T. Zier, N. Grigoryan, and M. E. Garcia, "Modeling of material properties after ultrashort laser and XUV excitation," Appl. Phys. A 110, 519-528 (2013).

${ }^{43}$ J. Zhang, C. Sun, B. Xiong, J. Wang, Z. Hao, L. Wang, Y. Han, H. Li, Y. Luo, Y. Xiao, C. Yu, T. Tanemura, Y. Nakano, S. Li, X. Cai, and S. Yu, "An InP-based vortex beam emitter with monolithically integrated laser," Nat. Commun. 9, 2652 (2018).

${ }^{44}$ L. E. Black, A. Cavalli, M. A. Verheijen, J. E. M. Haverkort, E. P. A. M. Bakkers, and W. M. M. Kessels, "Effective surface passivation of InP nanowires by atomic-layer-deposited $\mathrm{Al}_{2} \mathrm{O}_{3}$ with $\mathrm{PO}_{\mathrm{x}}$ interlayer," Nano Lett. 17, 6287-6294 (2017).

${ }^{45}$ W. S. Ko, I. Bhattacharya, T.-T. D. Tran, K. W. Ng, G. S. Adair, and C. Chang-Hasnain, "Ultrahigh responsivity-bandwidth product in a compact InP nanopillar phototransistor directly grown on silicon," Sci. Rep. 6, 33368 (2016).

${ }^{46}$ W.-Z. Xu, F.-F. Ren, D. Jevtics, A. Hurtado, L. Li, Q. Gao, J. Ye, F. Wang, B. Guilhabert, L. Fu, H. Lu, R. Zhang, H. H. Tan, M. D. Dawson, and C. Jagadish, "Vertically emitting indium phosphide nanowire lasers," Nano Lett. 18, 3414-3420 (2018)

${ }^{47}$ P. Ramasamy, N. Kim, Y.-S. Kang, O. Ramirez, and J.-S. Lee, “Tunable, bright, and narrow-band luminescence from colloidal indium phosphide quantum dots," Chem. Mater. 29, 6893-6899 (2017).

${ }^{48}$ P. Ramasamy, K.-J. Ko, J.-W. Kang, and J.-S. Lee, "Two-step "seed-mediated" synthetic approach to colloidal indium phosphide quantum dots with high-purity photo- and electroluminescence," Chem. Mater. 30, 3643-3647 (2018).

${ }^{49}$ J. P. Perdew, K. Burke, and M. Ernzerhof, "Generalized gradient approximation made simple,” Phys. Rev. Lett. 77, 3865-3868 (1996).

${ }^{50} \mathrm{~J}$. Heyd and G. E. Scuseria, "Assessment and validation of a screened Coulomb hybrid density functional,” J. Chem. Phys. 120, 7274-7280 (2004).

${ }^{51} \mathrm{~J}$. Heyd, G. E. Scuseria, and M. Ernzerhof, "Erratum: "Hybrid functionals based on a screened Coulomb potential" [J. Chem. Phys. 118, 8207 (2003)]," J. Chem. Phys. 124, 219906 (2006).
${ }^{52}$ J. P. Perdew, M. Ernzerhof, and K. Burke, "Rationale for mixing exact exchange with density functional approximations," J. Chem. Phys. 105, 9982-9985 (1996).

${ }^{53}$ S. L. Dudarev, G. A. Botton, S. Y. Savrasov, C. J. Humphreys, and A. P. Sutton, "Electron-energy-loss spectra and the structural stability of nickel oxide: An LSDA+U study," Phys. Rev. B 57, 1505-1509 (1998).

${ }^{54}$ P. E. Blöchl, "Projector augmented-wave method," Phys. Rev. B 50, 17953-17979 (1994).

${ }^{55} \mathrm{G}$. Kresse and D. Joubert, "From ultrasoft pseudopotentials to the projector augmented-wave method," Phys. Rev. B 59, 1758-1775 (1999).

${ }^{56} \mathrm{G}$. Kresse and J. Hafner, "Ab initio molecular dynamics for open-shell transition metals,” Phys. Rev. B 48, 13115-13118 (1993).

${ }^{57} \mathrm{G}$. Kresse and J. Furthmüller, "Efficient iterative schemes for ab initio totalenergy calculations using a plane-wave basis set," Phys. Rev. B 54, 11169-11186 (1996).

${ }^{58} \mathrm{P}$. Tangney and S. Fahy, "Density-functional theory approach to ultrafast laser excitation of semiconductors: Application to the $A_{1}$ phonon in tellurium," Phys. Rev. B 65, 054302 (2002).

${ }^{59}$ R. F. W. Bader, "Molecular fragments or chemical bonds," Acc. Chem. Res. 8, 34-40 (1975).

${ }^{60}$ R. Bader, Atoms in Molecules: A Quantum Theory, International Series of Monographs on Chemistry (Clarendon Press, 1994).

${ }^{61}$ B. Silvi and A. Savin, "Classification of chemical bonds based on topological analysis of electron localization functions," Nature 371, 683 (1994).

${ }^{62}$ A. Savin, R. Nesper, S. Wengert, and T. F. Fssler, "ELF: The electron localization function," Angew. Chem. 36, 1808-1832 (2003).

${ }^{63}$ A. D. Becke and K. E. Edgecombe, "A simple measure of electron localization in atomic and molecular systems," J. Chem. Phys. 92, 5397-5403 (1990).

${ }^{64}$ S. Baroni, S. de Gironcoli, A. Dal Corso, and P. Giannozzi, "Phonons and related crystal properties from density-functional perturbation theory," Rev. Mod. Phys. 73, 515-562 (2001).

${ }^{65} \mathrm{P}$. Giannozzi, S. de Gironcoli, P. Pavone, and S. Baroni, "Ab initio calculation of phonon dispersions in semiconductors," Phys. Rev. B 43, 7231-7242 (1991).

${ }^{66} \mathrm{G}$. Kresse, J. Furthmller, and J. Hafner, "Ab initio force constant approach to phonon dispersion relations of diamond and graphite," Europhys. Lett. 32, 729 (1995).

${ }^{67} \mathrm{~A}$. Togo, L. Chaput, and I. Tanaka, "Distributions of phonon lifetimes in Brillouin zones,” Phys. Rev. B 91, 094306 (2015).

${ }^{68} \mathrm{~A}$. Togo, F. Oba, and I. Tanaka, "First-principles calculations of the ferroelastic transition between rutile-type and $\mathrm{CaCl}_{2}$-type $\mathrm{SiO}_{2}$ at high pressures," Phys. Rev. B 78, 134106 (2008).

${ }^{69} \mathrm{~A}$. Togo and I. Tanaka, "First principles phonon calculations in materials science," Scr. Mater. 108, 1-5 (2015).

${ }^{{ }^{0}} \mathrm{H}$. Yao, L. Ouyang, and W.-Y. Ching, "Ab initio calculation of elastic constants of ceramic crystals,” J. Am. Ceram. Soc. 90, 3194-3204 (2007).

${ }^{71}$ D. Holec, M. Friák, J. Neugebauer, and P. H. Mayrhofer, "Trends in the elastic response of binary early transition metal nitrides," Phys. Rev. B 85, 064101 (2012).

${ }^{72} \mathrm{R}$. Yu, J. Zhu, and H. Ye, "Calculations of single-crystal elastic constants made simple," Comput. Phys. Commun. 181, 671-675 (2010).

${ }^{73}$ P. S. Branicio, J. P. Rino, C. K. Gan, and H. Tsuzuki, "Interaction potential for indium phosphide: A molecular dynamics and first-principles study of the elastic constants, generalized stacking fault and surface energies," J. Phys. Condens. Matter 21, 095002 (2009).

${ }^{74}$ A. Bouhemadou, R. Khenata, M. Kharoubi, T. Seddik, A. H. Reshak, and Y. Al-Douri, "FP-APW+lo calculations of the elastic properties in zinc-blende III-P compounds under pressure effects," Comput. Mater. Sci. 45, 474-479 (2009).

${ }^{75}$ C. M. O. Bastos, F. P. Sabino, G. M. Sipahi, and J. L. F. Da Silva, "A comprehensive study of g-factors, elastic, structural and electronic properties of III-V 
semiconductors using hybrid-density functional theory," J. Appl. Phys. 123, 065702 (2018).

${ }^{76}$ Z.-J. Wu, E.-J. Zhao, H.-P. Xiang, X.-F. Hao, X.-J. Liu, and J. Meng, "Crystal structures and elastic properties of superhard Ir $\mathrm{N}_{2}$ and Ir $\mathrm{N}_{3}$ from first principles," Phys. Rev. B 76, 054115 (2007).

${ }^{77}$ F. Mouhat and F. M. C.-X. Coudert, "Necessary and sufficient elastic stability conditions in various crystal systems," Phys. Rev. B 90, 224104 (2014).
${ }^{78}$ A. Debernardi, "Phonon linewidth in III-V semiconductors from densityfunctional perturbation theory,” Phys. Rev. B 57, 12847-12858 (1998).

${ }^{79}$ J. Fritsch, P. Pavone, and U. Schröder, "Ab initio calculation of the phonon dispersion in bulk InP and in the $\operatorname{InP}(110)$ surface," Phys. Rev. B 52, 11326-11334 (1995).

${ }^{80}$ P. H. Borcherds, G. F. Alfrey, A. D. B. Woods, and D. H. Saunderson, "Phonon dispersion curves in indium phosphide," J. Phys. C Solid State Phys. 8, 2022-2030 (1975). 